\title{
Prognostic Value of FDG-PET in Patients with Oropharyngeal Carcinoma Treated with Concurrent Chemoradiotherapy
}

Keisuke Enomoto, ${ }^{1,2}$ Hidenori Inohara, ${ }^{1}$ Ichiro Higuchi, ${ }^{2}$ Kenichiro Hamada, ${ }^{2}$ Yoichiro Tomiyama, ${ }^{1}$ Takeshi Kubo, ${ }^{1}$ Jun Hatazawa ${ }^{2}$

${ }^{1}$ Department of Otolaryngology, Osaka University School of Medicine, 2-2, Yamadaoka, Suita, Osaka 565-0871, Japan

${ }^{2}$ Department of Nuclear Medicine and Tracer Kinetics, Osaka University School of Medicine, 2-2, Yamadaoka, Suita, Osaka 565-0871, Japan

Tn Volume 10, Number 4 (July/August 2008), on page 225, in the "Materials and Methods" section, the second sentence of the subsection "Concurrent CRT" should read:

Chemotherapy consisted of six cycle of cisplatin $\left(20 \mathrm{mg} / \mathrm{m}^{2}\right)$ and docetaxel $\left(10 \mathrm{mg} / \mathrm{m}^{2}\right)$.

The authors regret the error.

The online version of the original article can be found at http://dx.doi.org/ $10.1007 / \mathrm{s} 11307-008-0145-4$ 